ACCEPTED For PUblication in ApJ, PART 1

Preprint typeset using $\mathrm{LAT}_{\mathrm{EX}}$ style emulateapj v. 20/04/00

\title{
THE JET AND CIRCUMBURST STELLAR WIND OF GRB $980519^{1}$
}

\author{
A. O. Jaunsen ${ }^{2,3}$, J. Huorth ${ }^{4,3,5}$, G. Buörnsson ${ }^{6}$, M. I. Andersen, ${ }^{7,8}$ H. Pedersen ${ }^{4}$,

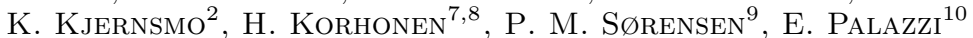 \\ Accepted for publication in ApJ, part 1
}

\begin{abstract}
We present extensive multi-colour $\left(U B V R_{C} I_{C}\right)$ photometry of the optical afterglow of GRB 980519 . Upon discovery, 8.3 hours after the burst, the source was decaying as a power law, $\left(t-t_{\mathrm{GRB}}\right)^{\alpha}$, with a rapid decay rate $\alpha_{1}=-1.73 \pm 0.04$. About 13 hours after the burst a steepening of the light-curve to $\alpha_{2}=-2.22 \pm 0.04$ was observed. Within the framework of current afterglow models, we argue that the rapid initial decline, the 'break' in the light curve, and the spectral properties of the afterglow are best interpreted as being due to a collimated ultra-relativistic jet of fixed opening angle expanding into an inhomogeneous medium. In this scenario, we find that the circumburst medium has a density structure that goes as $r^{-2.05 \pm 0.22}$. This is characteristic of a preexisting wind expelled from a massive star. A possible physical scenario is that the progenitor star collapsed to form a black hole (i.e., a 'collapsar'), producing the observed burst and afterglow. However, the supernova signature expected in the light curve in such a scenario is not detected. This either implies that the redshift of GRB 980519 is greater than 1.5 or that supernovae accompanying GRBs are not standard candles.
\end{abstract}

Subject headings: cosmology: miscellaneous - cosmology: observations - gamma rays: bursts supernovae: general - dust, extinction

\section{INTRODUCTION}

The nature of gamma-ray bursts (GRBs) has remained elusive for three decades. With their cosmological origin firmly established, the challenge now lies in identifying the GRB progenitors. Two types of candidates stand out as the currently most physically viable; the 'collapsing star' and 'merging binary compact object' models. These models can be constrained by the observed temporal evolution and spectral properties of the GRB afterglow.

The study of GRB afterglow light curves has shown that they are usually characterized by a power-law decay, $\left(t-t_{\mathrm{GRB}}\right)^{\alpha}$, as predicted in the generic "fireball" model in which the kinetic energy of ultra-relativisitc particles is converted to photons from a spatially small region (Piran 1999). A number of afterglows have been observed to decay at a very rapid rate. The decay rate, $\alpha$, depends on the nature of the fireball and also on the density structure of the ambient medium. There are currently two generic afterglow scenarios that result in steep light curves; the jet-model (Rhoads 1999; Sari, Piran, \& Halpern 1999) in which the relativistic outflow is collimated, and the wind model (Chevalier \& Li 1999) which invokes an inhomo- geneous circumburst medium. In a few cases the rate of decay has been observed to steepen, resulting in a 'break' in the light curve, which is most naturally accounted for by a collimated outflow.

Halpern et al. (1999) documented the steep power-law decay of GRB 980519 from a collection of own observations of the optical afterglow (OA) and data reported in circulars. The reported rapid decay, $\alpha=-2.05 \pm 0.04$, was interpreted in the context of both a spherical expansion into an inhomogeneous medium and a jet-model with lateral expansion in a homogeneous medium. On the basis of the available optical and X-ray data (in't Zand et al. 1999; Nicastro et al. 1999), Sari et al. (1999) advocated a jet-model while Chevalier \& Li (1999) advocated a windmodel. Frail et al. (1999) presented radio data which favor the wind-model, but could not exclude the jet-model. Further observations were reported by Vrba et al. (2000) and Smith et al. (1998) who presented optical and submillimeter observations of the OA, respectively. Finally, Sokolov et al. (1998) and Bloom et al. (1998b) independently detected an $R=26.1 \pm 0.3$ extended object at the approximate position of the OA in late July 1998, presumably the host galaxy.

\footnotetext{
${ }^{1}$ The Nordic Optical Telescope is operated on the island of La Palma jointly by Denmark, Finland, Iceland, Norway, and Sweden, in the Spanish Observatorio del Roque de los Muchachos of the Instituto de Astrofisica de Canarias. The data presented here have been taken using ALFOSC, which is owned by the Instituto de Astrofisica de Andalucia (IAA) and operated at the Nordic Optical Telescope under agreement between IAA and the Astronomical Observatory of the University of Copenhagen.

${ }^{2}$ Institute of Theoretical Astrophysics, University of Oslo, Pb. 1029, Blindern, N-0315 Oslo, Norway

${ }^{3}$ Centre for Advanced Study, Drammensvn. 78, N-0271 Oslo, Norway

${ }^{4}$ Astronomical Observatory, University of Copenhagen, Juliane Maries Vej 30, DK-2100 Copenhagen $\varnothing$, Denmark

${ }^{5}$ NORDITA, Blegdamsvej 17, DK-2100 Copenhagen $\varnothing$, Denmark

${ }^{6}$ Science Institute, University of Iceland, Dunhaga 3, IS-107 Reykjavik, Iceland

${ }^{7}$ Astronomy Division, Department of Physical Sciences, University of Oulu, FIN-90014 Oulu, Finland

${ }^{8}$ Nordic Optical Telescope, Ap. 474 St. Cruz de La Palma, E-38700 Canarias, Spain

${ }^{9}$ Isaac Newton Group, Santa Cruz de La Palma, E-38700 Canarias, Spain

${ }^{10}$ Instituto Tecnologie e Studio Radiazioni Extraterrestri, C.N.R., via Gobetti 101, 40129 Bologna, Italy
} 
Here we present $U B V R_{C} I_{C}$ photometry of the OA of GRB 980519. We find that the decay is not described by a single power law as previously suggested, but steepens about 13 hours after the burst. We discuss the implications of this result in the context of theoretical afterglow models.

\section{OBSERVATIONS}

The OA of GRB 980519 was discovered (Jaunsen et al. 1998; Hjorth et al. 1998) using the 2.56-m Nordic Optical Telescope (NOT) following a BATSE Rapid Burst Response on 1998 May 19.51403 UT (Muller 1998), and a subsequent localisation by the BeppoSAX Wide Field Cameras (WFC) to an error radius of 3' (Piro 1998). The first optical images were inspected and compared to a corresponding Digitized Sky Survey (DSS) ${ }^{11}$ image about 8.3 hours after the burst. The OA was confirmed to be fading by approximately $0.2 \mathrm{mag} /$ hour and was observed extensively during the night of discovery and at regular intervals the following days, weeks and months. Composite images of the fading OA are presented in Fig. 1.

\section{EDITOR: PLACE FIGURE 1 HERE.}

An astrometric plate solution computed relative to the USNO-A2.0 catalogue (Monet 1998) using the WCS-tools package (Mink 1999) gave $\alpha(J 2000)=$ $23^{\mathrm{h}} 22^{\mathrm{m}} 21^{\mathrm{s}} .55, \delta(J 2000)=77^{\circ} 15^{\prime} 43^{\prime \prime} \cdot 2$ for the OA.

The images were processed using standard tools in $I R A F^{12}$ and the $I_{C}$-band frames were also de-fringed using a master-fringe image constructed from all $I_{C}$-band science images. On 1998 May 24 we obtained multi-band imaging of the GRB-field and of the M92-field centered on the coordinates given in Christian et al. (1985) at three different airmasses for the purpose of independent photometric calibration. As reference we used a catalogue by P. Stetson (provided by F. Grundahl) calibrated to the Landolt-system and measured with PSF photometry. After matching and pruning the catalogue covering our subfield for close neighbors and large instrumental magnitude errors $(>0.02)$ we achieved a very good simultaneous fit of the zero-point, color-term and extinction. The RMS and the number of data points used in the fits were 0.023 (36), 0.032 (612), 0.022 (864), 0.025 (862) and 0.026 (553) for the $U, B, V, R_{C}$, and $I_{C}$ bands, respectively. This allowed us to photometrically calibrate several reference stars in the GRB field for all five bands to high precision. These measurements were made using SExtractor v.2.1.6 (Bertin \& Arnouts 1996) with fixed apertures plus aperture corrections extending to 15 arc-seconds. The M92 photometric calibration fit and the conversion from instrumental to absolute magnitudes was done using tools in the IRAF/PHOTCAL package. In Table 1 we give the results of the field photometry for all reference objects mentioned in relevant circulars (Jaunsen et al. 1998; Bloom et al. 1998a; Henden et al. 1998) using the same nomenclature (two additional objects introduced by A. Henden are also listed). Our absolute photometry of these point sources is in good agreement with that of Henden et al. (1998) and the robust average deviations are $0.03,0.03,0.01$, and 0.03 mag for the $B V R_{C} I_{C}$ bands. A systematic offset of 0.04 mag in the $R_{C}$-band is, however, found.

\section{EDITOR: PLACE TABLE 1 HERE.}

The OA was found to be consistent with a point source and we therefore chose to perform relative PSF photometry of the $\mathrm{OA}$ and a few neighboring stars to maximize the signal-to-noise. The PSF photometry measurements were done using DAOPHOT II (Stetson 1991). Absolute magnitudes were computed for each epoch from the average offset of the measured stars relative to the aperture measurements described above. The resulting photometry is listed in Table 2.

\section{EDITOR: PLACE TABLE 2 HERE.}

\section{THE LIGHTCURVE}

The magnitudes listed in Table 2 were converted to $\mu \mathrm{Jy}$ using the $\mathrm{AB}$ offsets and coefficients for the effective wavelengths of the respective passbands given in Fukugita, Shimasaku, \& Ichikawa (1995). The multi-colour light curves are depicted in Fig. 2.

\section{EDITOR: PLACE FIGURE 2 HERE.}

There were no obvious colour changes of the OA throughout the observing period. We observed a marked leveling off of the light curve in the $R_{C}$-band on 1998 May 30.00 UT. Such a behavior is usually ascribed to the constant contribution from a host galaxy, consistent with the detection of a faint, $R=26.1 \pm 0.3$, extended object (Sokolov et al. 1998; Bloom et al. 1998b). This host contribution was subtracted from the combined light curve in the lower panel of Fig. 2.

A power-law decay of the optical flux, $F_{\nu} \propto \nu^{-\beta} t^{\alpha}$, is expected in fireball models (Sari, Piran, \& Narayan 1998). Fitting a single power-law, including band offsets relative to the $R_{C}$-band was computed by weighted least chi-square minimization $\left(\chi_{25}^{2}=2.4\right.$, where the subscript represents the number of degrees of freedom in the fit). The errors for each component of the fit (including the band offset errors) are estimated as the perturbation needed to give a change in $\chi^{2}$ of 1 (corresponding to a 1-sigma error). The resulting power-law slope is $\alpha_{0}=-2.03 \pm 0.02$, consistent with that found by Halpern et al. (1999) and Vrba et al. (2000).

The combined multi-colour light curve, however, reveal the presence of a temporal break. A broken power-law fit $\left(\chi_{23}^{2}=0.26\right)$ to the data gives $\alpha_{1}=-1.73 \pm 0.04$ and $\alpha_{2}=-2.22 \pm 0.04$, with the break occurring at about $t_{\text {break }} \simeq 0.55$ days after the burst. The break point was estimated by extrapolating the two power-laws. The break

\footnotetext{
${ }^{11}$ The Digitized Sky Surveys were produced at the Space Telescope Science Institute under U.S. Government grant NAG W-2166. The images of these surveys are based on photographic data obtained using the Oschin Schmidt Telescope on Palomar Mountain and the UK Schmidt Telescope. The plates were processed into the present compressed digital form with the permission of these institutions.

${ }^{12}$ IRAF (Image Reduction and Analysis Facility) is distributed by the National Optical Astronomy Observatories, which are operated by the Association of Universities for Research in Astronomy, Inc., under contract with the National Science Foundation.
} 
in the light curve is highly significant. Taking the ratio of the two $\chi^{2}$ values yields $F_{23,25}=\chi_{25}^{2} / \chi_{23}^{2}=8.6$. Assuming the errors to be Gaussian distributed, $F_{23,25}$ can be approximated by a Fisher distribution (Lupton 1993). The broken power-law fit is therefore a significantly better representation of the light curve than the single power-law fit at the $99.99995 \%$ confidence level. The scatter in the combined multi-band light curve as compared to the broken power-law is $\lesssim 0.03$ mag. We also experimented with fitting a four-parameter function to the light-curve, similar to that used in eg. Stanek et al. (1999), but found the resulting errors much larger than the two power-law fit. We estimate the timescale on which the break occurs to be $\sim 0.2$ days (essentially the time difference between the adjacent end points on the two power laws in a given band).

The X-ray counterpart was observed by the BeppoSAX Wide Field Camera (WFC) up to about 130 seconds after the burst trigger. Follow-up Narrow Field Instruments (NFI) observations started 10 hours later. Nicastro et al. (1999) attempted to estimate the X-ray decay slope, $\alpha_{X}$, based on the WFC and NFI measurements, but noted that the NFI measurements do not seem to follow a simple power-law decay. We interpret their data as the signature of a break in the X-ray decay slope. The first NFI observation was made prior to the time of our estimated break, while the remaining NFI observations were made after the break. Consequently, the pre-break value of $\alpha_{X}$ can be estimated as the slope between the WFC and the first NFI measurement, giving approximately $\alpha_{X}=-1.6$. The post-break value of $\alpha_{X}$ is given approximately by the minimum estimate of Nicastro et al. (1999), $\alpha_{X}=-2.25 \pm 0.22$. The temporal break detected in the optical data is thus independently confirmed by the X-ray data.

\section{SPECTRAL PROPERTIES}

GRB afterglow is believed to be synchrotron emission by electrons accelerated at the relativistic shock between the fireball and the ambient medium, to a power-law energy distribution, $N(\gamma)=\gamma^{-p}$, where $\gamma$ is the electron Lorentz factor (Piran 1999). The resulting spectrum is then characterized by four power-law sections separated by three breaks at $\nu_{\mathrm{a}}, \nu_{\mathrm{m}}$ and $\nu_{\mathrm{c}}$ corresponding to the synchrotron self-absorption, peak energy and cooling frequencies, respectively (Sari et al. 1998). An optical broad-band spectrum coinciding in time with the first BeppoSAX NFI observation (Nicastro 1998) was constructed by interpolating (or mildly extrapolating) the flux in each band to a common date of May 19.96 UT.

Unfortunately, the reddening towards GRB 980519, $b^{I I} \sim 15^{\circ}$, is uncertain. The estimate of Schlegel, Finkbeiner, \& Davis (1998) for this region is $\mathrm{E}(\mathrm{B}-\mathrm{V})$ $=0.267$, but Arce \& Goodman (1999) argue that these reddening values are overestimated when $A_{V}>0.5$ (which is the case here). Since a small error in $\mathrm{E}(\mathrm{B}-\mathrm{V})$ translates into a large uncertainty in the inferred spectral slope, $\beta_{O}$, we refrain from estimating it directly from the broad-band spectrum. Instead, based on our data, we note that $\beta_{O}$ can be expressed as a linear function of the reddening as $\beta_{O} \simeq 3.32 \mathrm{E}(\mathrm{B}-\mathrm{V})-1.60$.

EDITOR: PLACE FIGURE 3 HERE.
Fig. 3 shows the optical data corrected for Galactic extinction using $\mathrm{E}(\mathrm{B}-\mathrm{V})=0.24$ (see discussion in Section 5) and the estimated X-ray spectral index (Nicastro et al. 1999), tied to the first NFI measurement. It is readily seen from both the uncorrected and extinction corrected optical data in Fig. 3, that the $B$ and to some extent $U$ values are inconsistent with a power-law. Assuming the spectral slope to be a power-law, the flux deficit may be due to extinction in the host galaxy, depletion by intergalactic Lyman- $\alpha$ absorption, or both. We therefore fit a power-law spectrum to the $V R I$-bands only.

The difference in the spectral indices of the optical and $\mathrm{X}$-ray regimes as discussed above and seen in Fig. 3 indicates the presence of a spectral break at approximately $\nu_{c} \simeq 2.3 \times 10^{16} \mathrm{~Hz}$. The steep initial temporal evolution of GRB 980519 and a spectral break between optical and $\mathrm{X}$-ray suggests emission by adiabatic electrons $\left(\nu_{\mathrm{m}}<\nu_{\mathrm{c}}\right)$.

\section{THE MODEL}

When the emitting electrons are adiabatic, it follows that the initial temporal evolution of the light-curve (whether the outflow is spherical or collimated) is given by $F_{\nu} \propto \nu^{-\beta} t^{-3 \beta / 2-\delta /(8-2 \delta)}$, with the ambient density profile of the form $n(r) \propto r^{-\delta}(\delta<3)$ (Mészáros, Rees, \& Wijers 1998; Panaitescu, Mészáros, \& Rees 1998). Here, $\delta=2$, represents a stellar wind density distribution, while $\delta=0$ represents a homogeneous density distribution. The temporal decay slope is thus steeper by $\delta /(8-2 \delta)$ in the inhomogeneous case.

If the ejecta is collimated, and the observer is within the collimated jet, the light curve is given by the above expression while there still is considerable relativistic beaming, i.e. while $\Gamma>1 / \theta$, where $\Gamma$ is the bulk Lorentz-factor and $\theta$ is the half angle of the collimated outflow. When the expansion slows down and $\Gamma$ drops below $1 / \theta$, the observer starts to see the edge of the jet and the light curve behavior switches to $F_{\nu} \propto \nu^{-\beta} t^{-3 \beta / 2-(6-\delta) /(8-2 \delta)}$. The light curve thus steepens by $\Delta \alpha=(3-\delta) /(4-\delta)$. An inhomogeneous environment therefore leads to a steeper light curve but a less pronounced break than in the homogeneous case. Specifically, a collimated outflow expanding into a circumstellar wind environment with $\delta=2$, would steepen by $1 / 2$, whereas the steepening in a homogeneous environment would be $3 / 4$.

In the case of GRB 980519 the existence of a temporal break is most naturally explained if the outflow was collimated. The steepening in the light curve is measured to be $\Delta \alpha=\alpha_{1}-\alpha_{2}=0.49 \pm 0.06$ which implies an environmental density gradient of $\delta=2.05 \pm 0.22$, in excellent agreement with the distribution expected from a circumstellar wind. The expected optical spectral index can be inferred from the light curve as $\beta=-2 / 3\left(\alpha_{1}+\delta /(8-2 \delta)\right)=0.80 \pm 0.08$. The relation between reddening due to Galactic extinction and $\beta_{O}$ given in Section 4 can then be used to infer $\mathrm{E}(\mathrm{B}-\mathrm{V})=0.24$ from the expected optical spectral index. This is the value used for the Galactic extinction correction of the optical data in Fig. 3 and is in good agreement with the value estimated from Schlegel et al. (1998). The power-law index of the electron energy distribution is $p=1+2 \beta=2.61 \pm 0.16$ (for $\nu_{\mathrm{m}}<\nu<\nu_{\mathrm{c}}$ ). The $\mathrm{X}$-ray spectral index then is $\beta_{X}=p / 2=1.30 \pm 0.08$ (for $\nu>\nu_{\mathrm{c}}$ ), consistent with the estimate of Nicastro et al. 
(1999), $\beta_{X}=1.8_{-0.5}^{+0.6}$, and that of Owens et al. (1998), $\beta_{X}=1.52_{-0.57}^{+0.7}$.

\section{DISCUSSION}

In the 'collapsar' scenario the gamma-ray burst and its afterglow are believed to be produced by a collapsing massive star (e.g., a Wolf-Rayet star) leading to a collimated ultra-relativistic outflow. The jet would then interact with an inhomogeneous density distribution created by a preexisting stellar wind expelled by the progenitor star (Fryer, Woosley, \& Hartmann 1999; MacFadyen \& Woosley 1999).

The identification of a spectral break between optical and X-rays, suggesting a fast cooling regime $\left(\nu>\nu_{\mathrm{c}}\right)$ at $\mathrm{X}$-ray frequencies, implies that the $\mathrm{X}$-ray decay slope is shallower by $1 / 4$ as compared to the optical (slow cooling) (Chevalier \& Li 1999). This is consistent with the temporal X-ray decay discussed in Section 3. The radio data presented by Frail et al. (1999) also appears to be consistent with the presented collapsar scenario. The collapsar model can thus account for the $\mathrm{X}$-ray, optical and radio observations of GRB 980519.

A potential problem for this interpretation concerns the sharpness of the observed break. The break in the light curve of GRB 980519 is rather sharply defined in time and occurred on a timescale of about 0.2 days. This is much shorter than expected from theoretical models. Moderski, Sikora, \& Bulik (2000) showed that the transition of the light curve from one asymptote to the other occurs within a factor of two in time for a jet of constant opening angle in a constant density environment. If the opening angle of the collimated outflow is allowed to evolve, the break becomes even smoother, i.e., extends over a longer period of time. Based on numerical simulations, Kumar \& Panaitescu (2000) argue that the transition in such cases takes place over a factor of at least 100 in time in a wind density environment. Much shorter break time scales, however, are expected for observers located on the jet axis.

In the collapsar scenario a re-brightening of the source $\sim 15(1+z)$ days after the burst is expected due to the onset of a supernova type Ibc explosion as energy is deposited by the jet (MacFadyen \& Woosley 1999). Bloom et al. (1999) noted that the rapid decay of the OA of GRB 980519 and faintness of the host makes it a good candidate for a SN detection. The expected late evolution based on the SN 1998bw light curves associated with GRB 980425 (Galama et al. 1998) are therefore given in Fig. 2 at various redshifts as in Bloom et al. (1999). We do not detect a re-brightening comparable to the SN 1998bw light curves. However, our last detection of the afterglow +host on 1998 May 30.00 UT, the subsequent upper limits and host detections by Sokolov et al. (1998); Bloom et al. (1998b) set a lower limit to the redshift of GRB 980519 of $z \gtrsim 1.5$ assuming the expected re-brightening is similar to SN 1998bw. This constraint on the redshift is consistent with the faintness of the host galaxy.

Alternatively, should the redshift of GRB 980519 turn out to be lower than 1.5, the absence of a SN signature in the data (see Fig. 2) can be taken as evidence that collapsars do not necessarily produce $\mathrm{SNe}$ of comparable brightness ('standard candle') to SN 1998bw (see Hjorth et al. 2000, suggesting this is the case in GRB 990712).

Finally, we note that Esin \& Blandford (2000) recently presented an alternative explanation for the excess red flux observed $\sim 20-30 \mathrm{~d}$ after the bursts of GRB 970228 (Fruchter et al. 1999) and GRB 980326 (Bloom et al. 1999), hitherto taken as evidence for the SNe interpretation and indirectly a 'collapsar' origin. Future observations should allow a discrimination between the two models.

\section{REFERENCES}

Arce, H. c. G. \& Goodman, A. A. 1999, ApJ, 512, L135

Bertin, E. \& Arnouts, S. 1996, A\&AS, 117, 393

Bloom, J. S., Djorgovski, S. G., Gal, R. R., Kulkarni, S. R., , \& Kelly, A. 1998a, GRB 980519 Optical Observations, GCN Circ. No. 87

Bloom, J. S., Kulkarni, S. R., Djorgovski, S. G., Eichelberger, A. C., Cote, P., Blakeslee, J. P., Odewahn, S. C., Harrison, F. A., et al. 1999, Nature, 401, 453

Bloom, J. S., Kulkarni, S. R., Djorgovski, S. G., Gal, R. R., Eichelberger, A., \& Frail, D. A. 1998b, GRB 980519, Optical observations of the host galaxy, GCN Circ. No. 149

Chevalier, R. A. \& Li, Z.-Y. 1999, Astrophys. J., 520, L29

Christian, C. A., Adams, M., Barnes, J. V., Butcher, H., Hayes, D. S., Mould, J. R., \& Siegel, M. 1985, Publ. Astron. Soc. Pac., 97,363

Esin, A. A. \& Blandford, R. 2000, ApJ, 534, L151

Frail, D. A., Kulkarni, S. R., Sari, R., Taylor, G. B., Shepherd, D. S., Bloom, J. S., Young, C. H., Nicastro, L., et al. 1999, Astrophys. J., submitted

Fruchter, A. S., Pian, E., Thorsett, S. E., Bergeron, L. E., González, R. A., Metzger, M., Goudfrooij, P., Sahu, K. C., et al. 1999, ApJ, 516,683

Fryer, C. L., Woosley, S. E., \& Hartmann, D. H. 1999, Astrophys. J., 526,152

Fukugita, M., Shimasaku, K., \& Ichikawa, T. 1995, Publ. Astron. Soc. Pac., 107, 945

Galama, T. J., Vreeswijk, P. M., van Paradijs, J., Kouveliotou, C., Augusteijn, T., Bohnhardt, H., Brewer, J. P., Doublier, V., et al 1998, Nature, 395, 670

Halpern, J. P., Kemp, J., Piran, T., \& Bershady, M. A. 1999, Astrophys. J., 517, L105
Henden, A. A., Vrba, F. J., Luginbuhl, C. B., Canzian, B., Levine, S. E., Guetter, H. H., \& Munn, J. A. 1998, GRB980519 Secondary Standards, GCN Circ. No. 96

Hjorth, J., Holland, S., Courbin, F., Dar, A., Olsen, L. F., \& Scodeggio, M. 2000, ApJ, 534, L147

Hjorth, J., Jaunsen, A. O., Kjernsmo, K., \& Pedersen, H. 1998, GRB 980519, Optical observations, GCN Circ. No. 84

in't Zand, J. J. M., Heise, J., van Paradjis, J., \& Fenimore, E. E. 1999, Astrophys. J., 516, L57

Jaunsen, A. O., Hjorth, J., Andersen, M. I., Kjernsmo, K., Pedersen, H., \& Palazzi, E. 1998, GRB 980519, Optical observations, GCN Circ. No. 78

Kumar, P. \& Panaitescu, A. 2000, astro-ph/0003264

Lupton, R. 1993, Statistics in Theory and Practice (Princeton University Press, Princeton, New Jersey)

MacFadyen, A. L. \& Woosley, S. E. 1999, Astrophys. J., 524, 262

Mészáros, P., Rees, M. J., \& Wijers, R. A. M. J. 1998, Astrophys. J., 499, 301

Mink, D. J. 1999, in Astronomical Data Analysis Software and Systems VIII, A.S.P. Conf. Series, ed. D. Mehringer, R. Plante, \& D. Roberts, 498

Moderski, R., Sikora, M., \& Bulik, T. 2000, Astrophys. J., 529, 151

Monet, D. 1998, flagstaff: USNO

Muller, J. M. 1998, GRB 980519, IAU Circ. No. 6910

Nicastro, L. 1998, GRB 980519, IAU Circ. No. 6912

Nicastro, L., Amati, L., Antonelli, L. A., Costa, E., Cusumano, G., Feroci, M., Frontera, F., Palazzi, E., et al. 1999, Astron. Astrophys. Suppl. Ser., 138, 437

Owens, A., Guainazzi, M., Oosterbroek, T., Orr, A., Parmar, A. N., Costa, E., Feroci, M., Piro, L., et al. 1998, A\&A, 339, L37

Panaitescu, A., Mészáros, P., \& Rees, M. J. 1998, Astrophys. J., 503,314

Piran, T. 1999, Phys. Rep., 314, 575 
Piro, L. 1998, GRB 980519, GCN Circ. No. 75

Rhoads, J. E. 1999, Astrophys. J., 525, 737

Sari, R., Piran, T., \& Halpern, J. P. 1999, Astrophys. J., 519, L17

Sari, R., Piran, T., \& Narayan, R. 1998, Astrophys. J., 497, L17

Schlegel, D. J., Finkbeiner, D. P., \& Davis, M. 1998, Astrophys. J. 500,525

Smith, I. A., Tilanus, R. P. J., van Paradijs, J., Galama, T. J. Groot, P. J., Vreeswijk, P., Kouveliotou, C., Wijers, R. A. M. J., et al. 1998, Astron. Astrophys., 347, 92
Sokolov, V., Zharikov, S., Palazzi, E., \& Nicastro, L. 1998, GRB 980519 Optical observations, GCN Circ. No. 148

Stanek, K. Z., Garnavich, P. M., Kaluzny, J., Pych, W., \& Thompson, I. 1999, ApJ, 522, L39

Stetson, P. B. 1991, AJ, 102, 932

Vrba, F. J., Henden, A. A., Canzian, B., Levine, S. E., Luginbuhl, C. B., Guetter, H. H., Munn, J. A., Hartmann, D. H., et al. 2000, ApJ, 528, 254

ACKNOWLEDGEMENTS. We thank L. Nicastro for contributing updated and refined X-ray values. AOJ thanks S. V. Haugan and O. Skjæraasen for helpful discussions. We thank the NOT Director for continued support to our GRB programme. This research was supported by the Danish Natural Science Research Council (SNF), the Icelandic Council of Science and the University of Iceland Research Fund. 
TABLE 1

$U B V R_{C} I_{C}$ FIELD PHOTOMETRY .

\begin{tabular}{|c|c|c|c|c|c|c|c|}
\hline ID & $\mathrm{RA}_{\mathrm{J} 2000}$ & $\mathrm{DEC}_{\mathrm{J} 2000}$ & $U$ & $B$ & $V$ & $R_{C}$ & $I_{C}$ \\
\hline $\mathrm{B}$ & $23: 22: 27.290$ & $+77: 15: 43.499$ & $22.78 \pm 0.11$ & $21.59 \pm 0.05$ & $20.21 \pm 0.02$ & $19.27 \pm 0.01$ & $18.46 \pm 0.01$ \\
\hline $\mathrm{E}$ & $23: 22: 20.201$ & $+77: 15: 24.001$ & $\ldots$ & & $21.15 \pm 0.04$ & $19.97 \pm 0.02$ & $18.63 \pm 0.02$ \\
\hline $\mathrm{F}$ & $23: 22: 31.399$ & $+77: 15: 43.301$ & & $22.63 \pm 0.12$ & $20.98 \pm 0.04$ & $19.81 \pm 0.02$ & $18.26 \pm 0.01$ \\
\hline $\mathrm{G}$ & $23: 22: 33.199$ & $+77: 14: 58.999$ & $22.74 \pm 0.10$ & $21.42 \pm 0.04$ & $19.88 \pm 0.02$ & $18.86 \pm 0.01$ & $17.93 \pm 0.01$ \\
\hline $\mathrm{J}$ & $23: 22: 07.250$ & $+77: 16: 00.599$ & $\ldots$ & $22.43 \pm 0.10$ & $20.93 \pm 0.03$ & $19.96 \pm 0.02$ & $19.14 \pm 0.02$ \\
\hline $\mathrm{M}$ & $23: 22: 46.610$ & $\begin{array}{r}7: 16: 43.399 \\
+\end{array}$ & $20.17 \pm 0.03$ & $19.10 \pm 0.01$ & $17.93 \pm 0.01$ & $17.21 \pm 0.00$ & $16.60 \pm 0.00$ \\
\hline $\mathrm{N}$ & $23: 22: 39.610$ & $+77: 12: 46.901$ & $\cdots$ & $16.74 \pm 0.01$ & $15.94 \pm 0.01$ & $15.45 \pm 0.01$ & $15.03 \pm 0.01$ \\
\hline
\end{tabular}

Note. - World coordinates computed relative to USNO A-2.0 (Monet 1998) using WCS-tools (Mink 1999) 
TABLE 2

OBSERVING LOG AND MAGNITUDES.

\begin{tabular}{lrrrr}
\hline \hline $\begin{array}{c}\text { Date } \\
1998 \text { May UT }\end{array}$ & $\begin{array}{c}\text { Exp. time } \\
\text { (sec) }\end{array}$ & Band & $\begin{array}{c}\text { Seeing } \\
\left.\text { FWHM }{ }^{\prime \prime}{ }^{\prime}\right)\end{array}$ & $\begin{array}{c}\text { Johnson-Cousins } \\
\text { Magnitude }\end{array}$ \\
\hline 19.861 & 60 & I & 1.20 & $18.42 \pm 0.09$ \\
19.864 & 180 & I & 1.33 & $18.47 \pm 0.05$ \\
19.877 & 300 & I & 1.27 & $18.52 \pm 0.02$ \\
19.933 & 500 & I & 1.79 & $18.83 \pm 0.04$ \\
20.002 & 500 & I & 1.51 & $19.09 \pm 0.02$ \\
20.047 & 500 & I & 1.49 & $19.26 \pm 0.02$ \\
20.136 & 500 & I & 1.02 & $19.60 \pm 0.02$ \\
20.210 & 500 & I & 0.92 & $19.89 \pm 0.02$ \\
20.976 & 500 & I & 1.39 & $21.71 \pm 0.20$ \\
21.164 & 600 & I & 1.21 & $21.88 \pm 0.11$ \\
21.889 & 1200 & I & 1.01 & $22.80 \pm 0.23$ \\
22.192 & 1200 & I & 1.02 & $23.28 \pm 0.18$ \\
19.887 & 300 & V & 1.97 & $19.64 \pm 0.02$ \\
19.926 & 300 & V & 2.40 & $20.01 \pm 0.04$ \\
19.940 & 500 & V & 2.35 & $19.99 \pm 0.03$ \\
19.993 & 500 & V & 1.99 & $20.22 \pm 0.02$ \\
20.040 & 500 & V & 1.62 & $20.36 \pm 0.02$ \\
20.129 & 500 & V & 1.32 & $20.67 \pm 0.02$ \\
20.203 & 500 & V & 1.11 & $20.98 \pm 0.02$ \\
20.230 & 450 & V & 1.50 & $20.90 \pm 0.10$ \\
20.969 & 500 & V & 1.53 & $22.68 \pm 0.12$ \\
21.156 & 500 & V & 1.03 & $23.05 \pm 0.11$ \\
20.054 & 500 & R & 1.52 & $19.84 \pm 0.01$ \\
20.151 & 500 & R & 1.03 & $20.27 \pm 0.01$ \\
20.223 & 300 & R & 1.09 & $20.48 \pm 0.03$ \\
23.190 & 5100 & R & 0.84 & $24.31 \pm 0.12$ \\
30.003 & 5600 & R & 0.62 & $25.74 \pm 0.25$ \\
20.061 & 500 & B & 1.71 & $21.12 \pm 0.02$ \\
20.143 & 500 & B & 1.38 & $21.50 \pm 0.03$ \\
20.217 & 500 & B & 1.26 & $21.83 \pm 0.05$ \\
20.069 & 600 & U & 1.78 & $20.75 \pm 0.07$ \\
\hline & & & &
\end{tabular}


FIG. 1. - True-colour images centered on the fading optical counterpart of GRB 980519 at epoch 1998 May 20.05, 20.136 and 20.21 UT. North is up and East is to the left. The field measures $1^{\prime} \times 1^{\prime}$.

FIG. 2. - The upper panel shows the multi-colour light curves of GRB 980519 with the expected re-brightening due to an SN explosion based on the SN 1998bw light curves (Galama et al. 1998). The host detections by Sokolov et al. (1998) and Bloom et al. (1998b) are marked by boxes. The lower panel shows the combined data points after the offsets relative to the $R_{C}$-band data have been applied and the host contribution subtracted. The interpolated (solid lines) and extrapolated (dotted lines) power-laws are also plotted. The inset shows the data from the first night only.

FIG. 3.- In the left panel we show the broad-band spectrum before (filled) and after (open) correction for Galactic extinction. The right panel shows the corrected and extrapolated optical spectral slope and the X-ray measurement by Nicastro et al. (1999) with the estimated X-ray slope corresponding to $\beta_{X}=1.8_{-0.5}^{+0.6}$ (dashed) and its 1-sigma uncertainty values tied by the X-ray measurement (dotted). 


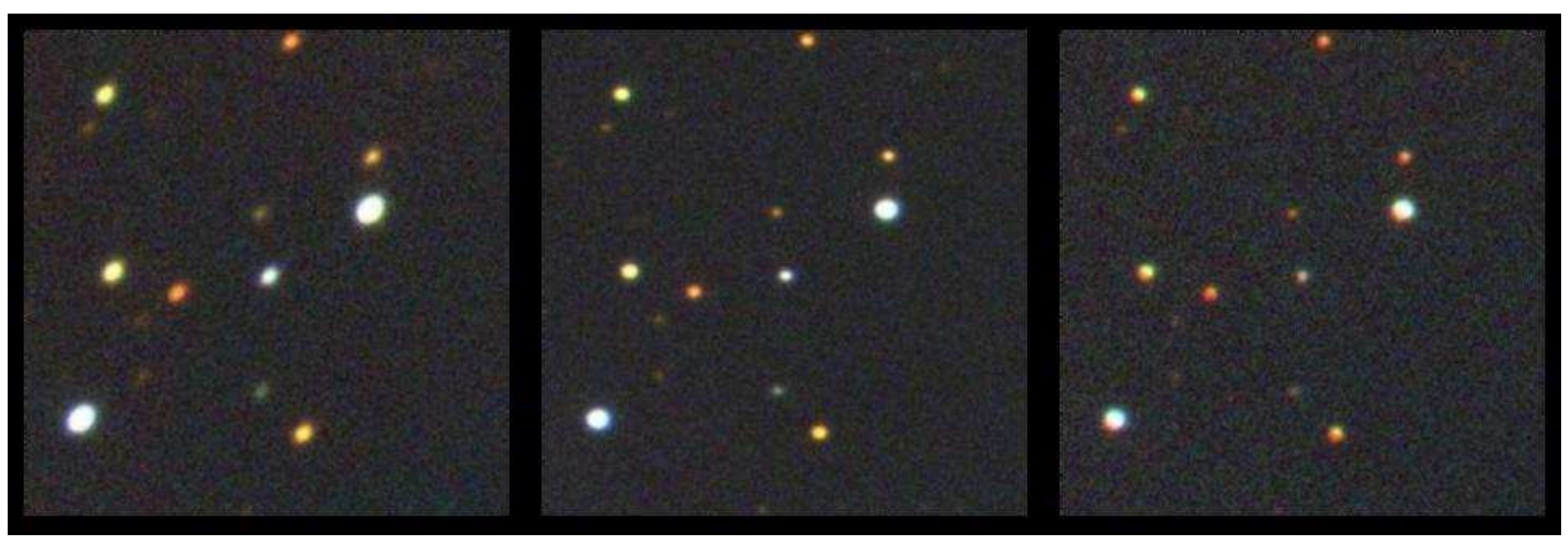




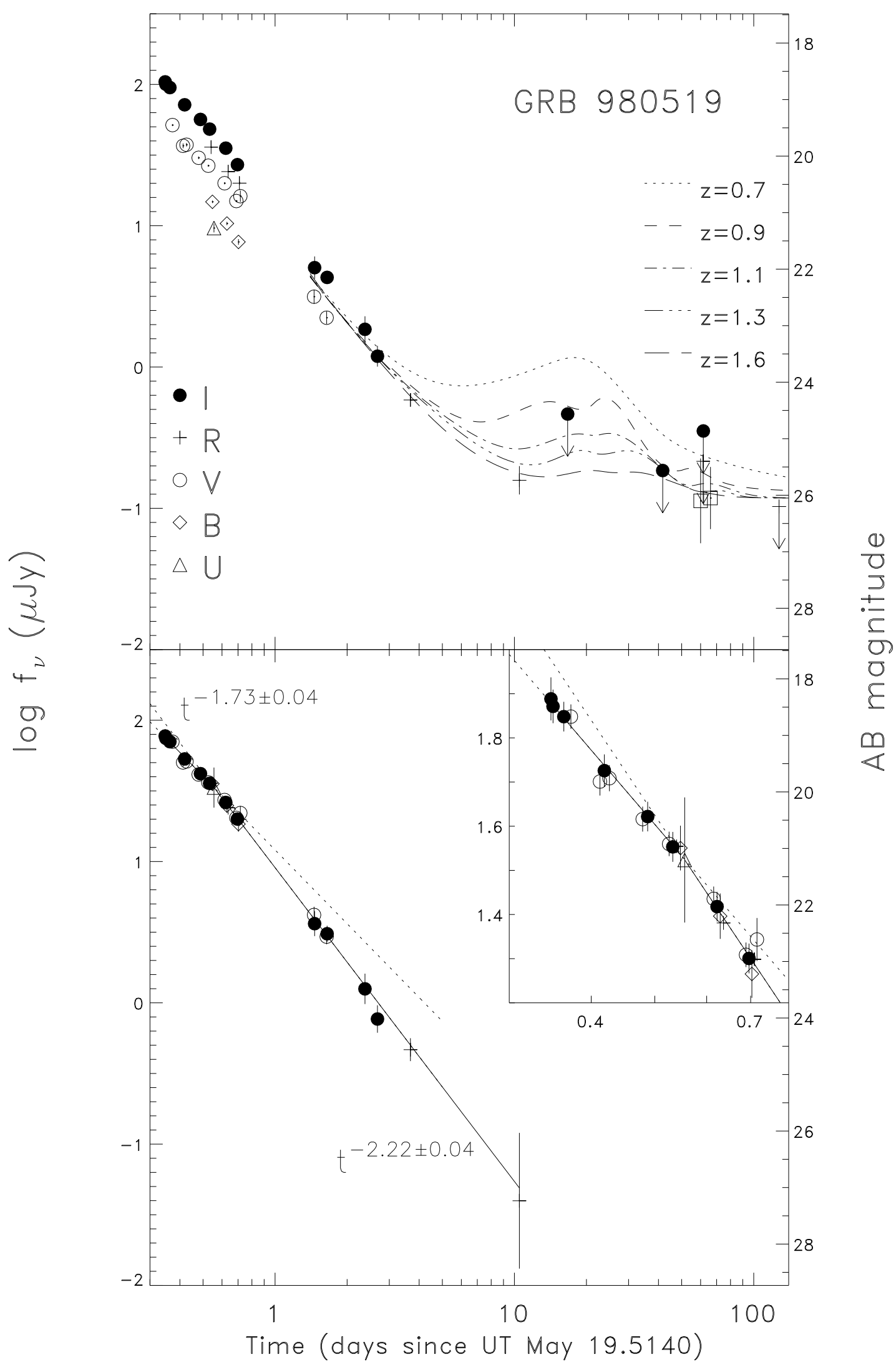



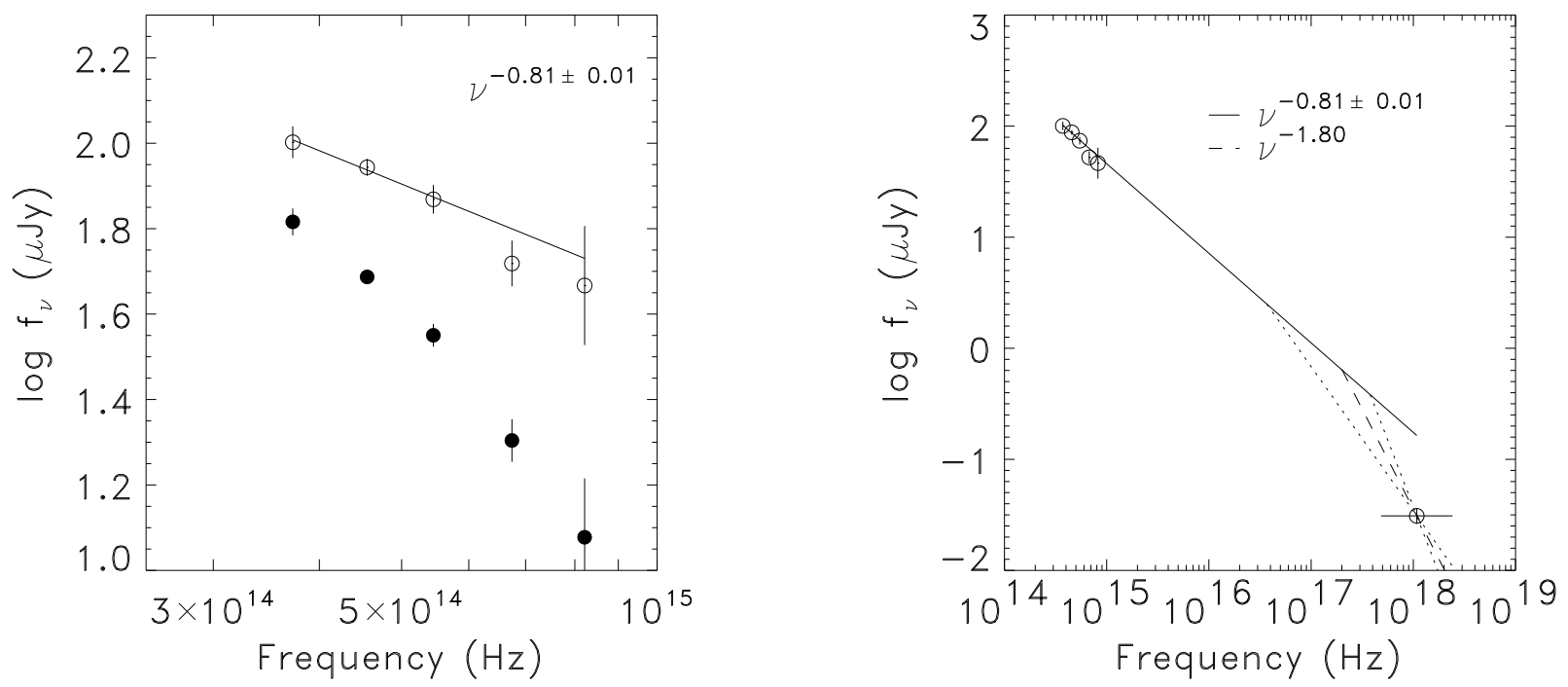